\title{
THERAPEUTIC MR IMAGE SEGMENTATION BASED ON UPGRADED FUZZY CLUSTERING ALGORITHM WITH BIAS FIELD ESTIMATION
}

\author{
L. Sathish Kumar \\ Department of Computer Science, Ananda College, India
}

\begin{abstract}
Therapeutic MR image segmentation is difficult in medical image processing. There are huge of issue are come about in the actual world medical images. In this research paper, gives method bias field estimation based fuzzy clustering technique. Scan corrupted and saltand-paper noise using Bias field estimation. Easy and simple to classify a given medical image database over a certain number of cluster fixed a-priori technique. In this research article, segmentation and Bias field estimation of brain MR images and involved the fuzzy clustering algorithm. In new improved technique evaluates the ability of Fuzzy cMean to segment White and Gary matter. It delivers extra prospective for efficiently segmenting MRI data and time consuming. The Gaussian weights is explore the delivery of the feature vectors in the scan image clusters. The empirical evaluation UFCA and fuzzy clustering, with Bias field estimation is achieved.
\end{abstract}

Keywords:

Bias Field Estimation, Fuzzy C Means, Improved Fuzzy Clustering Algorithm

\section{INTRODUCTION}

Therapeutic imaging of internal parts of the human body is significant to increase medical diagnosis and psychoanalysis. Medical image segmentation is a major mission in Therapeutic imaging. In line for to weak enhanced contrast and poor resolution this task is difficult in the presence of artifacts and noise [1]. Several existing approaches for segmentation are based on image shape, properties intensity information or shape prior [1] - [5] Propose multi-organ abdominal segmentation with analyses of missing organs using statistical location classical. In [6], the author presents instantaneous extraction of many organs from abdominal CT using stomach cavity regularization process with atlas guided segmentation incorporating parameter estimation and medical image database for organ separation. The method in [7] describes multi-region separation using graph cut method for four stomach organ segmentation. The method in [8] proposes a multi organ segmentation from MRI and CT medical images using shape representation and learning-based segmentation. In [9], the author described multi-organ segmentation based on classified spatial demonstrating of organ interrelations using medical images. Medical Image segmentation is the most thoughtprovoking and challenging issues in system idea usually and therapeutic imaging solicitations predominantly. Separation dividers image area or bulk into none overlapping, being homogeneous and connected regions with respect to some signal individualities [10]. The segmentation methods are subject to various tasks stemming from image noise, image artifacts such as discontinuities of edges, boundaries due to similar visual appearance of adjacent brain structures and partial volume effect. A variation of segmentation approaches methods has been developed to address these dares. Human Brain MR Image segmentation approaches can be categorized into three main categories: probabilistic, atlas-based, statistical-based and deformable model-based methods.

\section{RELATED WORKS}

Medical MRI Image segmentation plays a critical role in numerous bio medical MRI imaging presentations, health care professionals assisting technicians during the diagnosis of various illness diseases. A new fuzzy level set procedure is explore in this research paper to facilitate therapeutic image segmentation which is able to straight change from the initial separation of spatial fuzzy clustering. The Three-dimensional induced fuzzy c-means using pixel classification is applying vigorous variation boundaries for medical image segmentation. The monitoring parameters of level set advancement are also projected from the results of clustering. The fuzzy level set procedure is enhanced with locally standardized advancement. Such as developments simplify level set manipulation and lead to more integration. Enactment evaluation of the proposed approach was approved on medical MRI images from altered modalities. The author [11] examined the enactments FCM, k-Means, C-Means. Both detachment measures such as Euclidean (ED) and Manhattan $(\mathrm{MH})$ are used to note how these distance measures are influence the complete clustering enactment. The enactment has been associated based on seven constraints, sensitivity, accuracy, specificity, execution time, correctness, and average, inter cluster distance and intra cluster distance. Based on the empirical results, the research paper decided that three methods k-Means and FCM and C-Means performed well.

\section{PROPOSED UFCA METHOD}

The Fuzzy clustering algorithm based on Medical MRI image strength is done by the preliminary segmentation. Which trails the via field estimation technique for object modification by tracking the border variation the extensively used conservative fuzzy Cmeans for medical image segmentation is shaped norm distance quantity to measure the similarity between data object of medical image and center which are cheap by the weighty noise outliers and other imaging article.

The main objective of our approach is fuzzy clustering method and bias field estimation. The Fuzzy clustering plays role an imperative role in resolving issues in the areas of fuzzy model identification and outline recognition. A dissimilar types of fuzzy clustering methods has been projected and most of them are distance criteria based methods upon [12]. The Bias field estimation was Useful in scan corrupted by salt and paper noise one widely used procedure is the fuzzy c-means (FCM) procedure. Segmentation and Bias field estimation the edge from 
Time consuming and blurring it is uses reciprocal distance to compute fuzzy weights. The recommended method for improved fuzzy clustering the algorithms were customary for segmentation. The proposed technique is done by Filtering, Noises are reduced, Used improved fuzzy c-means algorithms which are very correct to detect the tumour affected area, and it shrinks the time for analysis.

\subsection{EXISTING FUZZY C MEANS CLUSTERING}

Fuzzy c-means (FCM) is a technique of clustering [5] which permits one sector of data to belong to more than clusters. The clustering algorithm was initially presented by Dunn and followed this algorithm extended by Bezdek. The FCM algorithm is an iterative clustering technique that gives an optimal c divider by weighted within group sum of squared error objective is minimized.

$$
Y_{m}=\sum_{i=1}^{N} \sum_{j=1}^{C} u_{i j}^{m}\left\|X_{i}-X_{j}\right\|^{2}
$$

where, $A=\left\{A_{1}, A_{2}, \ldots, a_{n}\right\}$ is the set of data in the $p$-dimensional vector space, number of data items is $n, c$ is represented by number of clusters with $2 \leq c<n, u_{i k}$ dimension degree of association of $x_{k}$ in the $i^{\text {th }}$ cluster, weighting exponent of $q$, every fuzzy membership $v_{i}$ is the prototype of the center of the cluster distance measure between object $x_{k}$ and cluster center $v_{i}$ of $i d^{2}$.

\subsection{PROPOSED UFCA CLUSTERING ALGORITHM}

UFCA Upgrade Fuzzy Clustering Algorithm is which allow segmented by more clusters. The UFCA algorithm intensity with decently to fuzzy membership is $U$ and $V$ is means set of clusters.

$$
J_{m}(U, V)=\sum_{i=1}^{N} \sum_{j=1}^{m} U_{i j}^{q}\left(x_{i}, \varnothing_{j}\right)
$$

The number of clusters $m$ is represented and number of feature vectors is $n$. Here this method, assumed in the number of clusters is four, that is namely white matter, background, gray matter, background belong to similar class and outcome, reduces to three number of classes. The UFCA algorithm is performed to objective function is minimized, herewith admiration to fuzzy membership $u_{i j}$ for $j^{\text {th }}$ cluster centroids $\theta_{j}$ and feature vector $x_{i}$ to $j^{\text {th }}$ cluster.

Here $B=\left\{b_{1}, b_{2}, b_{j}, \ldots, b_{N}\right\} p \times N$ is a data matrix, every $x_{j}$ vector is represents $p$ the dimension, and the number of feature vectors (pixel numbers in the image) represents $N$. number of clusters is represented $C$. The membership function of vector $x_{j}$ to the $i^{\text {th }}$ cluster is represented by $U_{i j} \subseteq U(p, N, C)$ which $u_{i j} \in[0,1]$ satisfies.

The tissue classes of mean intensity for initializing both UFCA and k-Means is derived from a histogram method. In the histogram funneled initialization, vector of mean intensity of $k$ is $\beta$, pre-processed image is used by tissue process,

$$
\beta=\left\{\beta_{1}, \beta_{2}, \beta_{3}, \ldots, \beta_{k}\right\}
$$

and $j$ is arbitrary sequence

$$
j=\{0,1,2,3, \ldots, k\}
$$

The pixel intensities range is the interval between maximum and minimum intensities in the pre-processed MRI image is separated into $k$ concentration bins, with $k+1$ greatness points between the minimum and maximum intensity. The MRI image formation with multiplicative additive noise and bias:

$$
I=b J+n
$$

where, $I$ is measured for image intensity, $J$ is used for stored true signal, bias field is represented in $b$, and additive noise is $n$. The main aim of bias dishonored is to estimate the bias field $b$ from the measured intensity $I$. the proposed approach Upgrade fuzzy Cluster algorithm (UFCA) and dissimilarity function is followed

$$
\varnothing_{j}=\frac{\sum_{i=1}^{m} U_{i j}^{u} x_{i}}{\sum_{i=1}^{N} U_{i j}^{q}}
$$

Here $\left(a_{j}, b_{j}\right)$ location of $j^{\text {th }}$ pixels. Two parameters magnitudes of $\lambda$ and $\zeta$ are between $[0,1]$; modify the degree of the two community pulls. For any defining using an ANN, objective function, input image constant parameters $\lambda$ and $\xi$ are calculable.

\section{EMPIRICAL FINDINGS AND RESULTS}

The proposed method UFCA is focus to bias correction and clustered of brain MRI images. These images exhibit obvious intensity in consistencies. The Fig. 1 shows the segmentation results attributes of computed number of iteration and results of existing and K-means, UFCA, and finally execution time in the second, third, and four column respectively. Taken input MRI scan image for .bmp format involve into the developing platform Matlab

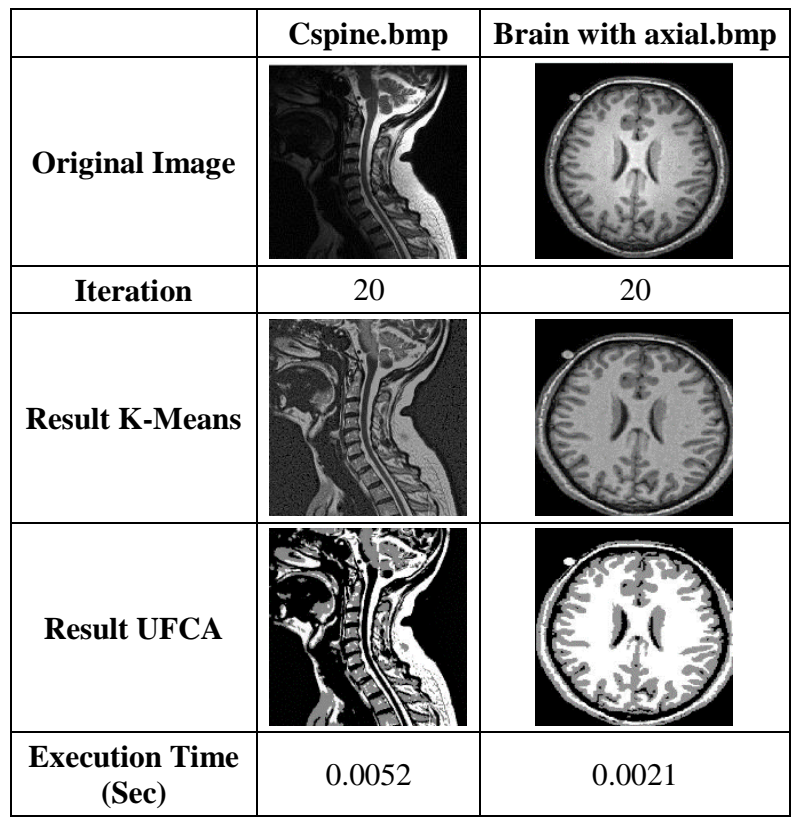

Fig.1. Comparison of existing and proposed methods result to an MR image of Brain and Cspine

\begin{tabular}{|c|c|c|}
\hline & Cspine.bmp & Brain with axial.bmp \\
\hline $\begin{array}{c}\text { Original } \\
\text { Image }\end{array}$ & & \\
\hline
\end{tabular}




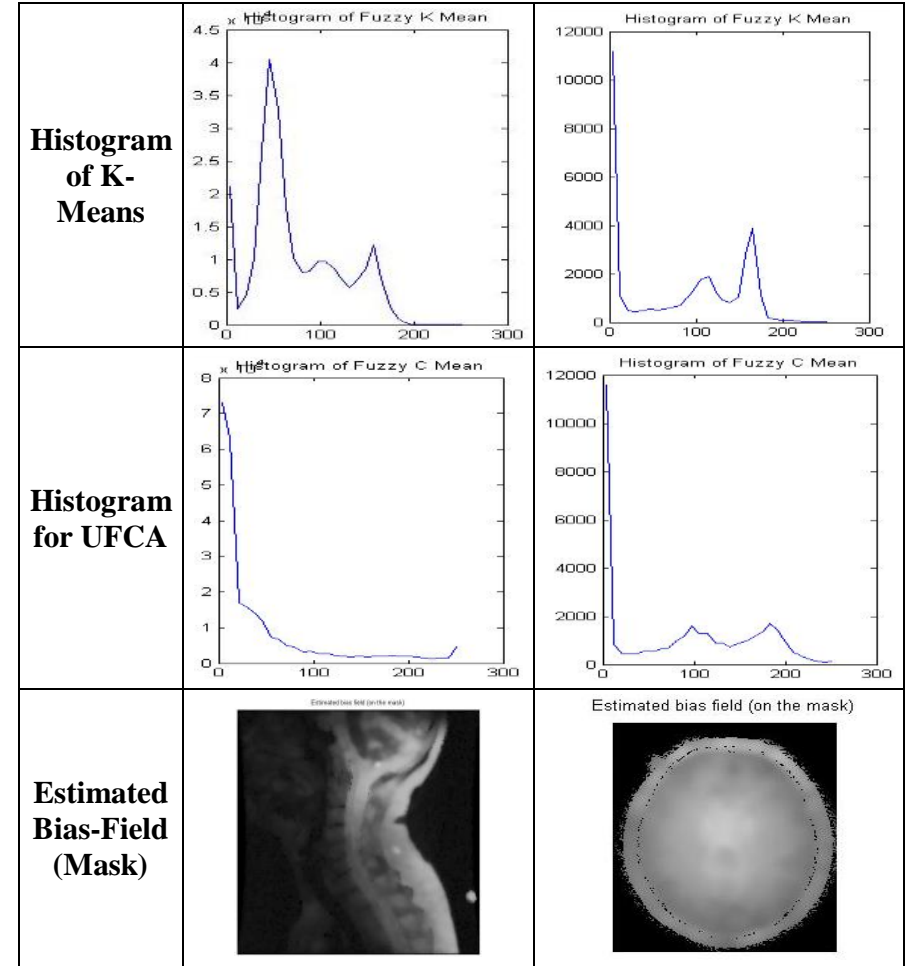

Fig.2. UFCA method to an MR image of Brain and Cspine

That UFCA algorithm can be seen corrected images with quite homogeneous. The development homogeneity is demonstrated by comparing the iteration and performance of results and execution time by iteration. The existing method result image in third column, proposed method result in the 4th column and execution time is last column in the Fig.1. The number of iteration, and results of proposed methods parameters are indicate the proposed method is best one of compared with existing method. The improved intensity of homogeneity demonstrate by comparing the existing image histogram with proposed method result image histogram and the estimated bias-field for the proposed method demonstrate in to the Fig.2. In Fig.2, well-defined and wellsegmented mountains in the histograms of the bias modified image, every conforming to a tissue or the upbringing in the MRI taken image. The proposed method histogram and Bias-field extraction score good performance compared with existing method.

\section{CONCLUSIONS}

The propose UFCA use fuzzy clustering method in Therapeutic MR image segmentation using bias field estimation for fuzzy clustering. The UFCA algorithm used reciprocal distance to calculate the fuzzy weights. The segmentation and Bias field estimation is used in scan corrupted by salt and paper and other noise MR images. It gave more potential for efficiently segmenting MR Image data and time consuming for execution. The Gaussian weights replicate the distribution of the feature vectors in the MR image clusters. Many empirical using both general images and medical image prove the advantages of UFCA method. In the proposed UFCA method has been involved to MR medical scan image with respective results.

\section{REFERENCES}

[1] Shawn Andrews, Chris McIntosh and Ghassan Hamarneh, "Convex Multiregion Probabilistic Segmentation with Shape Prior in Isometric Log-Ratio transformation Space", IEEE International Conference on Computer Vision, pp. 2096-2099, 2011.

[2] T. Chan and L. Vese, "Active Contours without Edges", IEEE Transactions on Image Processing, Vol. 10, No. 2, pp. 266-277, 2001.

[3] F. Rathke, S. Schmidt and C. Schnorr, "Order Preserving and Shape Prior Constrained Intra-retinal Layer Segmentation in Optical Coherence Tomography", Medical Image Computing and Computer-Assisted Intervention, Vol. 14, No. 3, pp. 370-377, 2011.

[4] B.N. Li, C.K. Chui, S. Chang and S.H. Ong, "Integrating Spatial Fuzzy Clustering With Level Set Methods For Automated Medical Image Segmentation”, Computer in Biology and Medicine, Vol. 41, No. 1, pp. 1-11, 2011.

[5] Miyuki Suzuki, Marius George Linguraru, Ronald M. Summers and Kazunori Okada, "Analyses of Missing Organs in Abdominal Multi-Organ Segmentation”, Proceedings of International MICCAI Workshop on Computational and Clinical Challenges in Abdominal Imaging, pp. 256-263, 2011.

[6] A. Shimizu, R. Ohno, T. Ikegami, H. Kobatake, S. Nawano and D. Smutek, "Segmentation of Multiple Organs in NonContrast 3D Abdominal CT Images", International Journal of Computer Assisted Radiology and Surgery, Vol. 2, No. 34, pp. 135-142, 2007.

[7] M.G. Linguraru, J.A. Pura, A.S. Chowdhury and R.M. Summers, "Multi-Organ Segmentation from Multi-Phase Abdominal CT Via 4D Graphs using Enhancement, Shape and Location Optimization", Medical Image Computing and Computer-Assisted Intervention, Vol. 13, No. 3, pp. 89-96, 2010.

[8] T. Kohlberger, M. Sofka, J. Zhang, N. Birkbeck, J. Wetzl, J. Kaftan, J. Declerck and S.K. Zhou, "Automatic Multi-Organ Segmentation using Learning- Based Segmentation and Level Set Optimization", Proceedings of International Conference on Medical Image Computing and ComputerAssisted Intervention, pp. 338-345, 2011.

[9] T. Okada, M.G. Linguraru, Y.Yoshida, M. Hori, R.M. Summers, Y.W. Chen, N. Tomiyama and Y. Sato, "Abdominal Multi-Organ Segmentation of CT Images Based on Hierarchical Spatial Modeling of Organ Interrelations", Proceedings of International MICCAI Workshop on Computational and Clinical Challenges in Abdominal Imaging, pp. 173-180, 2011.

[10] Ahmed Elnakib, Georgy Gimel'farb, Jasjit Suri and Ayman El-Baz, "Medical Image Segmentation: A Brief Survey", Available at: http://citeseerx.ist.psu.edu/viewdoc/download?doi=10.1.1.3 96.7312\&rep=rep1\&type=pdf.

[11] Q. Wen, M.E. Celebi, "Hard Versus Fuzzy c-Means Clustering for Color Quantization", Journal on Advances in Signal Processing, Vol. 118, pp. 1-6, 2011.

[12] Lawrence C. Evans, "Partial Differential Equations", $2^{\text {nd }}$ Edition, American Mathematical Society, 1998. 